\title{
Solution of some parabolic inverse problems by homotopy analysis method
}

\author{
O.N.Onyejekwe \\ Department of Mathematics, Eastern Florida State College, USA \\ *Corresponding author E-mail: onyejekweo@easternflorida.edu
}

Copyright $\odot 2014$ O.N.Onyejekwe. This is an open access article distributed under the Creative Commons Attribution License, which permits unrestricted use, distribution, and reproduction in any medium, provided the original work is properly cited.

\begin{abstract}
In this paper, three types of parabolic inverse problems are solved by homotopy analysis method (HAM). In order to solve these types of problems, an overspecified boundary condition is given. There are advantages to using HAM, firstly it is independent of small/large physical parameters, there is always a guarantee of convergence; there is flexibility on the choice of base function and initial guess of solution and lastly there is great generality. The numerical results obtained from this method indicate high accuracy and a strong rate of convergence.
\end{abstract}

Keywords: Control Function, Homotopy Analysis Method, One-Dimensional Heat Equation, Parabolic Inverse Problem.

\section{Introduction}

Most problems that arise in science and engineering are nonlinear in nature and are modeled with differential equations. We employ the use of approximate analytical methods to solve problems whose solutions are not so easily obtained analytically. In recent years many analytical methods have been developed; some of the methods employed are the homotopy analysis method [1], [10], [11], [15], [16], the homotopy perturbation method [13], [14], variational iteration method [13] and the adomain decomposition method [2], [3], [4], [11]. We utilize the homotopy analysis method (HAM) proposed by Liao $[15,16]$ to help us obtain exact and approximate solutions to inverse problems.

Unlike other perturbation methods, HAM avoids discretization, provides us with efficient numerical solution with high accuracy; there is minimal calculation and the avoidance of physically unrealistic assumptions. The convergence region for the series solution obtained by HAM is determined by the auxiliary parameter $\eta$.

In this paper, the Homotopy Analysis Method will be used to obtain an approximate analytic solution to a space dependent source term, a time dependent source term, a non-homogeneous unknown control function and the determination of the source function for a nonlinear parabolic heat equation.

When we solve inverse problems we determine or predict model parameters in this case the source term from the knowledge of the measured parameters or data. A lot of work and research has been done in solving inverse problems to nonlinear partial differential equations, some of the work may be found in [2], [5], [6], [8], [9], [11], [14], [18], and [19].

This article contains the following sections:

In section 2 we discuss the methodology of the Homotopy analysis method. We look at the solution of parabolic inverse problems with unknown control function in section 3. At the end of section 3, we discuss and analyze the results.

\section{Homotopy analysis method}

To illustrate the basic idea of the HAM, we consider the following differential equation:

$N[u(x, t)]=s(x, t)$, 
Where $\mathrm{N}$ is a nonlinear operator, $\mathrm{x}$ and $\mathrm{t}$ denote independent and dependent variables respectively, $\mathrm{u}$ is an unknown function and $\mathrm{s}(\mathrm{x}, \mathrm{t})$ is the nonhomogeneous term. By means of HAM, we first construct a zeroth-order deformation equation

$(1-q) L\left[\varphi(x, t ; q)-u_{0}(x, t)\right]=q \mathrm{~h} N[\varphi(x, t ; q)-s(x, t)]$,

Where $q \in[0,1]$ is the embedding parameter, $\mathrm{h} \neq 0$ is an auxiliary parameter, $\mathrm{L}$ is an auxiliary linear operator, $\varphi(x, t ; q)$ is an unknown factor, $u_{0}(x, t)$ is an initial guess of $\mathrm{u}(\mathrm{x}, \mathrm{t})$. It is obvious that when the embedding parameter $\mathrm{q}$ goes from 0 to 1 , the values for $\varphi(x, t ; q)$ becomes

$\varphi(x, t ; 0)=u_{0}(x, t), \quad \varphi(x, t ; 1)=u(x, t)$,

Respectively. Thus as q increases from 0 to 1 , the solution $\varphi(x, t ; q)$ varies from the initial guess $u_{0}(x, t)$ to the solution $\mathrm{u}(\mathrm{x}, \mathrm{t})$. Expanding $\varphi(x, t ; q)$ in Taylor series with respect to $\mathrm{q}$, we obtain

$\varphi(x, t ; q)=u_{0}(x, t)+\sum_{m=1}^{+\infty} u_{m}(x, t) q^{m}$

Where

$u_{m}(x, t)=\left.\frac{1}{m !} \frac{\partial^{m} \varphi(x, t ; q)}{\partial q^{m}}\right|_{q=0}$,

The convergence of the series (4) depends upon the auxiliary parameter $h$.

With HAM, we have the freedom to choose the initial guess $u_{0}(x, t)$, the auxiliary linear operator L, the nonzero auxiliary parameter $\mathrm{h}$ and the auxiliary function $\mathrm{H}(\mathrm{x}, \mathrm{t})$. We assume that all of them are properly chosen so that:

1) The solution $\varphi(x, t ; q)$ of the zeroth-order deformation equation (2) exists for all $q \in[0,1]$.

2) The homotopy analysis derivative $D_{m}(\varphi(x, t ; q))$ exists for $\mathrm{m}=1,2,3, \ldots,+\infty$.

3) The power series (4) of $\varphi(x, t ; q)$ converges at $\mathrm{q}=1$.

Then from Eqs. (2.3) and (2.4), we have under these assumptions the solution series

$u(x, t)=u_{0}(x, t)+\sum_{m=1}^{+\infty} u_{m}(x, t)$

Which must be one of the solutions of the original nonlinear equation, as proven by Liao [16]. Define the vectors

$\overrightarrow{u_{n}}=\left\{u_{0}(x, t), u_{1}(x, t), \ldots, u_{n}(x, t)\right\}$,

Differentiating the zeroth-order deformation equation (2.2) $\mathrm{m}$ - times with respect to $\mathrm{q}$ and then dividing them by $\mathrm{m}$ ! And finally setting $\mathrm{q}=0$ (Taking the $\mathrm{mth}$ - order homotopy derivative). Firstly, since $\mathrm{L}$ is a linear operator independent of q, it holds

$D_{m}\left((1-q) L\left[\varphi(x, t ; q)-u_{0}(x, t)\right]\right)$

$=D_{m}\left(L\left[\varphi(x, t ; q)-q \varphi(x, t ; q)+u_{0}(x, t) q-u_{0}(x, t)\right]\right)$,

And using Homotopy Properties (see Appendix) (i) and (ii)

$D_{m}\left(L\left[\varphi(x, t ; q)-q \varphi(x, t ; q)+u_{0}(x, t) q-u_{0}(x, t)\right]\right)$

$=L\left[D_{m} \varphi(x, t ; q)-D_{m}\left(q \varphi(x, t ; q)+u_{0} D_{m}(q)\right)\right]$,

And Homotopy Property (iii) and (IV)

$L\left[D_{m}(\varphi(x, t ; q))-D_{m}(q \varphi(x, t ; q))+u_{0}(x, t) D_{m}(q)\right]$

$=L\left[u_{m}(x, t)-u_{m-1}(x, t)+u_{0}(x, t) D_{m}(q)\right]$,

Which equals the $L\left[x_{m}\right]$ when $\mathrm{m}=1$, and $L\left[x_{m}-x_{m-1}\right]$ when $\mathrm{m}>1$, respectively. Thus, the $\mathrm{m}$ th - order deformation equation becomes

$L\left[u_{m}(x, t)-\chi_{m} u_{m-1}(x, t)\right]=\mathrm{h} R_{m}\left(\begin{array}{c}u_{m-1} \\ )\end{array}\right.$

Where

$R_{m}\left(\vec{u}_{m-1}\right)=\left.\frac{1}{(m-1) !} \frac{\partial^{m-1} N[\varphi(x, t ; q)-g(x, t)]}{\partial q^{m-1}}\right|_{q=0}$,

And 
$\chi_{m}=\left\{\begin{array}{l}0, m \leq 1 \\ 1, m>1\end{array}\right.$,

It should be emphasized that $u_{m}(x, t)$ for $m \geq 1$ is governed by the linear equation (11) with linear boundary conditions that come from the original problem. Therefore the solution to the differential equation obtained by HAM is a family of solutions expressed using the auxiliary parameter $\mathrm{h}$.

\section{Inverse parabolic problems with unknown control function}

In this paper, we use HAM to obtain an approximate analytical solution to the following inverse problem of finding both the control parameter $\mathrm{p}=\mathrm{p}(\mathrm{t})$ and $\mathrm{u}(\mathrm{x}, \mathrm{t})$ in the quasilinear time-dependent diffusion equation.

$\frac{\partial u}{\partial t}=\frac{\partial^{2} u}{\partial x^{2}}+p(t) u(x, t)+z(x, t): 0 \leq x \leq l, 0 \leq t \leq T_{0}$,

With initial condition and boundary conditions:

$u(x, 0)=f(x), u(0, t)=g_{0}(t), u(l, t)=g_{1}(t) ; 0 \leq t \leq T_{0}, 0 \leq x \leq l$,

The over specified boundary condition is

$u\left(x_{1}, t\right)=k_{1}(t) ; \quad 0<x_{1}<1,0 \leq t \leq T_{0}$,

All the conditions in (3.2) and (3.3) are known, $x_{1}, T_{0}$ and $l$ are positive numbers. Our aim is to solve for the unknowns, mainly the temperature $u(x, t)$ and the control function $p(t)$. Equation (3.1) is used to describe a heat converting process with source parameters and equation (3.3) displays the temperature at point $x_{1}$ at time t. The purpose of this paper is to determine the control parameters that are used to find the temperature $\mathrm{u}(\mathrm{x}, \mathrm{t})$ at any time $\mathrm{t}$ at the given point $x_{1}$.

The existence and uniqueness of (3.1)-(3.3) has been proved in references [5], [6], [7], [8], [11].

By using the conversion

$v(x, t)=r(t) u(x, t) ; r(t)=e^{-\int_{0}^{t} p(s) d s}$,

Equation (3.1) to (3.3) becomes as follows:

$v_{t}=v_{x x}+r(t) z(x, t) ; 0 \leq x \leq l, 0<t<T_{0}$,

$v(x, 0)=f(x), v(0, t)=r(t) g_{0}(t), v(l, t)=r(t) g_{1}(t) ; 0 \leq x \leq l, 0<t<T_{0}$,

Where we have:

$r(t)=\frac{v\left(x_{1}, t\right)}{k_{1}(t)} ; \quad 0<t \leq T_{0}$,

And

$p(t)=-\frac{r^{\prime}(t)}{r(t)} ; \quad 0<t \leq T_{0}$,

To solve (3.5) by HAM

Using the solution procedure by HAM, we define a linear operator in the form

$L[\phi(x, t ; q)]=\frac{\partial \phi(x, t ; q)}{\partial t}$,

With the property

$L\left[c_{1}(x)\right]=0$,

Where $c_{1}(x)$ is the integration constant the nonlinear operator is taken as

$N[\phi(x, t ; q)]=-v_{t}(x, t ; q)+v_{x x}(x, t ; q)+r(t) z(x, t)$,

So we can define $R_{m}$ as

$R_{m}\left(\vec{v}_{m-1}\right)=-\frac{\partial v_{m-1}(x, t)}{\partial t}+\frac{\partial^{2} v_{m-1}(x, t)}{\partial x^{2}}+r_{m-1}(t) z(x, t)$,

Using (2.11),(2.13) and (3.10), we can get

$v_{m}(x, t)=\chi_{m} v_{m-1}(x, t)+\mathrm{h} \int R_{m}\left(\vec{v}_{m-1}\right) d t+c_{1}(x)$,

The parameters used during the application of HAM are defined as follows: 
The initial guess is

$v_{0}(x, t)=u(x, 0)=f(x)$,

The calculations for $r(t), u(x, t)$ and $\mathrm{p}(\mathrm{t})$ is as follows:

$$
\begin{aligned}
& r(t)=\sum_{m=0}^{+\infty} r_{m}(t)=\sum_{m=0}^{+\infty} \frac{v_{m}(0, t)}{g_{1}(t)}=r_{0}+r_{1}+r_{2}+\ldots, \\
& u(x, t)=\sum_{m=0}^{+\infty} u_{m}(x, t)=\sum_{m=0}^{+\infty} \frac{v_{m}(x, t)}{r_{m}(t)}=u_{0}+u_{1}+u_{2}+\ldots, \\
& p(t)=\sum_{m=0}^{+\infty} p_{m}(t)=\sum_{m=0}^{+\infty} \frac{\left(u_{m}(x, t)\right)_{t}-\left(u_{m}(x, t)\right)_{x x}-z(x, t)}{u_{m}(x, t)}=p_{0}+p_{1}+p_{2}+\ldots,
\end{aligned}
$$

\section{Example 3.1:}

$z(x, t)=e^{t}\left[x+\cos (\pi x)+\pi^{2} \cos (\pi x)\right]-e^{t}\left(1+t^{2}\right)[x+\cos (\pi x)]$,

$g_{1}(t)=e^{t}$,

$k_{1}(t)=\frac{1}{2} e^{t}$, note $x_{1}=\frac{1}{2}$,

$f(x)=\cos (\pi x)+x$,

For which the exact solution is

$u(x, t)=e^{t}[\cos (\pi x)+x]$

And

$p(t)=1+t^{2}$

The results obtained for $\mathrm{u}(\mathrm{x}, \mathrm{t})$ and $\mathrm{p}(\mathrm{t})$ computed for $\mathrm{h}=0.0, T_{0}=1.0$ using HAM are listed below

Table 1: Results for u with $\mathrm{h}=0, T_{0}=1.0$

\begin{tabular}{llll}
\hline $\mathrm{x}$ & Exact $\mathrm{u}$ & HAM & Absolute Error \\
\hline 0.0 & 2.718281828 & 2.718281828 & 0 \\
0.05 & 2.820729359 & 2.820729359 & 0 \\
0.10 & 2.857067829 & 2.857067829 & 0 \\
0.15 & 2.829749117 & 2.829749117 & 0 \\
0.20 & 2.742792560 & 2.742792560 & 0 \\
0.25 & 2.601685971 & 2.601685971 & 0 \\
0.30 & 2.413250518 & 2.413250518 & 0 \\
0.35 & 2.185472765 & 2.185472765 & 0 \\
0.40 & 1.927308010 & 1.92308010 & 0 \\
0.45 & 1.648459787 & 1.648459787 & 0 \\
0.50 & 1.359140914 & 1.359140914 & 0 \\
0.55 & 1.069822040 & 1.069822040 & 0 \\
0.60 & 0.7909738171 & 0.7909738171 & 0 \\
0.65 & 0.532809062 & 0.532809062 & 0 \\
0.70 & 0.3050313084 & 0.3050313084 & 0 \\
0.75 & 0.1165958575 & 0.1165958575 & 0 \\
0.80 & -0.02451073229 & -0.02451073229 & 0 \\
0.85 & -0.1114672901 & -0.1114672901 & 0 \\
0.90 & -0.1387860010 & -0.1387860010 & 0 \\
0.95 & -0.1024475314 & -0.1024475314 & 0 \\
1.0 & 0 & 0 & 0 \\
\hline
\end{tabular}

\section{Example 3.2:}

$$
\begin{aligned}
& z(x, t)=\left(\pi^{2}-(t+1)^{2}\right) e^{-t^{2}}(\cos (\pi x)+\sin (\pi x)) \\
& g_{1}(t)=e^{-t^{2}} \\
& g_{2}(t)=-\pi e^{-t^{2}} \\
& k_{1}(t)=-e^{-t^{2}}, \text { note } x_{1}=1
\end{aligned}
$$


$f(x)=\cos (\pi x)+\sin (\pi x)$

For which the exact solution is

$u(x, t)=e^{-t^{2}}[\cos (\pi x)+\sin (\pi x)]$

And

$p(t)=1+t^{2}$

The results obtained for $\mathrm{u}(\mathrm{x}, \mathrm{t})$ and $\mathrm{p}(\mathrm{t})$ using HAM are listed below

Table 2: Results for $\mathrm{p}$ with $\mathrm{h}=0$

\begin{tabular}{|c|c|c|c|}
\hline $\mathrm{t}$ & Exact $p$ & HAM & Absolute Error \\
\hline 0.0 & 1 & 1 & 0 \\
\hline 0.05 & 1.002500000 & 1.002500000 & 0 \\
\hline 0.10 & 1.010000000 & 1.010000000 & 0 \\
\hline 0.15 & 1.022500000 & 1.022500000 & 0 \\
\hline 0.20 & 1.040000000 & 1.040000000 & 0 \\
\hline 0.25 & 1.062500000 & 1.062500000 & 0 \\
\hline 0.30 & 1.090000000 & 1.090000000 & 0 \\
\hline 0.35 & 1.122500000 & 1.122500000 & 0 \\
\hline 0.40 & 1.160000000 & 1.160000000 & 0 \\
\hline 0.45 & 1.202500000 & 1.202500000 & 0 \\
\hline 0.50 & 1.250000000 & 1.250000000 & 0 \\
\hline 0.55 & 1.302500000 & 1.302500000 & 0 \\
\hline 0.60 & 1.360000000 & 1.360000000 & 0 \\
\hline 0.65 & 1.422500000 & 1.422500000 & 0 \\
\hline 0.70 & 1.490000000 & 1.490000000 & 0 \\
\hline 0.75 & 1.562500000 & 1.562500000 & 0 \\
\hline 0.80 & 1.640000000 & 1.640000000 & 0 \\
\hline 0.85 & 1.722500000 & 1.722500000 & 0 \\
\hline 0.90 & 1.810000000 & 1.810000000 & 0 \\
\hline 0.95 & 1.902500000 & 1.902500000 & 0 \\
\hline 1.0 & 2.000000000 & 2.000000000 & 0 \\
\hline
\end{tabular}

Table 3: Results for $\mathrm{u}$, with $\mathrm{h}=0, T_{0}=0.25$

\begin{tabular}{llll}
\hline $\mathrm{x}$ & Exact $\mathrm{u}$ & HAM & Absolute Error \\
\hline 0.0 & 0.9394130628 & 0.9394130628 & 0 \\
0.05 & 1.0748039090 & 1.0748039090 & 0 \\
0.10 & 1.1837295160 & 1.1837295160 & 0 \\
0.15 & 1.2635077740 & 1.2635077740 & 0 \\
0.20 & 1.3121742770 & 1.3121742770 & 0 \\
0.25 & 1.3285306940 & 1.3285306940 & 0 \\
0.30 & 1.3121742770 & 1.3121742770 & 0 \\
0.35 & 1.2635077740 & 1.2635077740 & 0 \\
0.40 & 1.1837295160 & 1.1837295160 & 0 \\
0.45 & 1.0748039090 & 1.0748039090 & 0 \\
0.50 & 0.9394130628 & 0.9394130628 & 0 \\
0.55 & 0.7808907485 & 0.7808907485 & 0 \\
0.60 & 0.6031403139 & 0.6031403139 & 0 \\
0.65 & 0.4105385618 & 0.4105385618 & 0 \\
0.70 & 0.2078279878 & 0.2078279878 & 0 \\
0.75 & 0.0000000001 & 0.0000000001 & 0 \\
0.80 & -0.2078279887 & -0.2078279887 & 0 \\
0.85 & -0.4105385627 & -0.4105385627 & 0 \\
0.90 & -0.6031403147 & -0.6031403147 & 0 \\
0.95 & -0.7808907492 & -0.7808907492 & 0 \\
1.0 & -0.9394130628 & -0.9394130628 & \\
\hline
\end{tabular}


Table 4: Results for $\mathrm{p}$ with $\mathrm{h}=0$

\begin{tabular}{llll}
\hline $\mathrm{t}$ & Exact $\mathrm{p}$ & HAM & Absolute Error \\
\hline 0.0 & 1 & 1 & 0 \\
0.05 & 1.002500000 & 1.002500000 & 0 \\
0.10 & 1.010000000 & 1.010000000 & 0 \\
0.15 & 1.022500000 & 1.022500000 & 0 \\
0.20 & 1.040000000 & 1.040000000 & 0 \\
0.25 & 1.062500000 & 1.062500000 & 0 \\
0.30 & 1.090000000 & 1.090000000 & 0 \\
0.35 & 1.122500000 & 1.122500000 & 0.000000001 \\
0.40 & 1.160000000 & 1.159999990 & 0 \\
0.45 & 1.202500000 & 1.202500000 & 0 \\
0.50 & 1.250000000 & 1.250000000 & 0 \\
0.55 & 1.302500000 & 1.302500000 & 0 \\
0.60 & 1.360000000 & 1.360000000 & 0 \\
0.65 & 1.422500000 & 1.422500000 & 0 \\
0.70 & 1.490000000 & 1.490000000 & 0.000000001 \\
0.75 & 1.562500000 & 1.562500001 & 0 \\
0.80 & 1.640000000 & 1.640000000 & 0 \\
0.85 & 1.722500000 & 1.722500000 & 0 \\
0.90 & 1.810000000 & 1.810000000 & 0.000000001 \\
0.95 & 1.902500000 & 1.902500001 & 0.000000001 \\
1.0 & 2.000000000 & 2.000000001 & \\
\hline
\end{tabular}

\section{Conclusion}

We have shown that HAM can be used to accurately predict the results for the temperature and the control function. The solution series obtained for analytical approximation for $\mathrm{u}(\mathrm{x}, \mathrm{t})$ and $\mathrm{p}(\mathrm{t})$ contained the auxiliary term $\mathrm{h}$. Instead of using the $\mathrm{h}$-curve proposed by Liao [16], to determine the values of $\mathrm{h}$, we used the over specified boundary condition The freedom of choice in choosing $\mathrm{h}$ enables us to adjust and control the convergence of the solutions series and this differentiates the homotopy analysis method from other existing methods such as the homotopy perturbation method, variational iteration method and Adomian decomposition method.

MAPLE has been used for the computation in this paper.

\section{Acknowledgements}

The author would like to thank the referees for their constructive suggestions and valuable comments.

\section{APPENDIX}

Homotopy Properties (HP): For homotopy-series

$$
\hat{u}=\sum_{i=0}^{+\infty} u_{i}(\hat{v}) q^{i}, \quad \hat{v}=\sum_{i=0}^{+\infty} v_{i}(t) q^{i}
$$

The following properties are satisfied:

i. Let $\mathrm{f}$ and $\mathrm{g}$ be functions independent of the homotopy - parameter $\mathrm{q}$, then

ii. $\quad D_{m}(f \hat{u}+g \hat{v})=f D_{m}(\hat{u})+g D_{m}(\hat{v}) \cdot \mathrm{u}$

iii. Let $\mathrm{L}$ be a linear operator independent of the homotopy - parameter $\mathrm{q}$, then

iv. $\quad D_{m}(\hat{L} \hat{u})=L D_{m}(\hat{v})$.

v. $\quad D_{m}(\hat{u})=u_{m}$.

vi. $\quad D_{m}\left(q^{k} \hat{u}\right)=D_{m-k}(\hat{u})$.

vii. If $\hat{u}=\hat{v}$ in a domain $q \in[0, a]$, then $D_{m}(\hat{u})=D_{m}(\hat{v})$, thus $u_{m}=v_{m}$.

For proof of these properties, see Liao [17]. 


\section{References}

[1] A.K. Alomari, "Modifications of Homotopy Analysis Method for Differential Equations: Modification of Homotopy analysis method, ordinary, fractional, delay and algebraic differential equations”, Lambert Academic Publishing, Germany, 2012.

[2] A.M. Shahrezaee, "Solution of Some Parabolic Inverse Problems by Adomian Decomposition Method", Applied Mathematical Sciences, 5 (2011), 3949 -3958.

[3] A.M.Wazwaz, "Partial Differential Equations and Solitary Waves Theory", Springer, Berlin Heidelberg, 2009.

[4] A.M.Wazwaz, "Linear and Nonlinear Integral Equations", Springer, Berlin Heidelberg, 2011.

[5] E.G. Savateev, R. Riganti, "Inverse Problem for the Nonlinear Heat Equation with the Final Overdetermination", Mathl. Comput. Modelling, 22 (1995), $29-43$.

[6] J.R.Cannon, Y.Lin, "Numerical Solutions of Some Parabolic Inverse Problems", Numer. Math. Partial Diff. Eqns, $2(1990) 177$-191.

[7] J.R.Cannon, H.M Yin, "On a class of non-classical parabolic problems", J. Diff Eqs, 79 (1989), 266 -288.

[8] J.R.Cannon, Y. Lin and S. Wang, "Determination of source parameter in parabolic equations", Meccanica, 27 (1992), 85 -94.

[9] J.R.Cannon, Y.Lin, "An inverse problem of finding a parameter in a semilinear heat equation", J.Math.Anal.Appl, (1945), 470-484.

[10] K. Hosseini, B. Daneshian, N. Amanifard and R. Ansari, "Homotopy Analysis Method for a Fin with Temperature Dependent Internal Heat Generation and Thermal Conductivity”, International Journal of Nonlinear Science, 14 (2012), 201 - 210.

[11] M.Aylin Bayrak, Iclal Ulvi , "Identifying an Unknown Function in a Parabolic Equation by Homotopy Analysis Method and Comparison with the Adomian Decomposition Method", First International Conference on Analysis and Applied Mathematics, 1470 (2012), 88 - 91.

[12] M. Dehghan, "Parameter Determination in a Partial Differential Equation from the Overspecified Data", Mathematical and Computer Modelling, 41(2005), 197-213.

[13] M.Mousa, "Modifications of Homotopy perturbation and variational iteration methods: Convergence theorems and applications in fluid mechanics and mathematical physics", Lambert Academic Publishing, Germany, 2011.

[14] S.Aminsadrabad, "Solution for Inverse Space - Dependent Heat Source Problems by Homotopy Perturbation Method", Applied Mathematical Sciences, 6 (2012), $575-578$.

[15] S. Liao, "Homotopy Analysis Method in Nonlinear Equations", Springer, New York, 2012.

[16] S. Liao, "Beyond Perturbation: Introduction to the Homotopy Analysis Method", Chapman \& Hall/CRC, 2004.

[17] S. Liao, "Notes on the homotopy analysis method - Some definitions and theorems", Commun. Nonlinear Sci. Numer. Simulat, 14(2009), 983997.

[18] S. Wang and Y.Lin, "A finite difference solution to an inverse problems determining a control function in a parabolic partial differential equation", Inverse Problems,(1989) $631-640$.

[19] X. Li , S. Qian, "Numerical Solution of the Inverse Problem of Determining an Unknown Source Term in a Heat Equation", Hindawi Publishing Corporation, Journal of Applied Mathematics, http://dx.doi:10.1155/2012/390876. 